\title{
STABILIZING FIRST-ORDER CONTROLLERS WITH DESIRED STABILITY REGION
}

\author{
K. Saadaoui* and A.B. Özgüler**
}

\begin{abstract}
In this paper, we determine the set of all stabilizing first-order controllers that place the poles of the closed-loop system in a desired stability region. The solution is based on a generalization of the Hermite-Biehler theorem applicable to polynomials with complex coefficients and the application of a modified stabilizing gain algorithm to three subsidiary plants. The method given is also applicable to PID controllers.
\end{abstract}

\section{Key Words}

Hermite-Biehler theorem, stabilization, first-order controllers, regional pole placement

\section{Introduction}

In many applications, stability of the closed-loop system is not enough, and it is usually required that the poles of the closed-loop system lie in more restrictive stability regions. It is known that time domain specifications for a closed-loop system can be translated into desired closedloop poles locations in the frequency domain. These are specified in terms of the damping ratio and damped natural frequency of the closed-loop poles [1]. A desired stability region $S$ in the complex plane is shown in Fig. 1, [2]. The region $S$ is the intersection of three regions $S_{-\lambda}, S_{\theta}$, and $S_{-\theta}$ where $S_{-\lambda}:=\{s: s \in \mathbf{C}, \operatorname{Re}[s]<-\lambda\}$ is a shifted Hurwitz stability region, $S_{\theta}:=\left\{s: s \in \mathbf{C}, \operatorname{Re}\left[s e^{-j \theta}\right]<0\right\}$ and $S_{-\theta}:=\left\{s: s \in \mathbf{C}, \operatorname{Re}\left[s e^{j \theta}\right]<0\right\}$ are rotated Hurwitz stability regions. In [3], it is stated that if all the poles of the closed-loop system lie in the region $S$, then the step response of the compensated system exhibits a settling time of no more than $4 / \lambda$ and a maximum overshoot corresponding to the angle $\theta$. In [4], the region $S$ is approximated by a circular region and a design procedure that combines linear-quadratic optimal control with

* Unité de recherche LA.R.A. Automatique, Ecole Nationale d'Ingénieurs de Tunis, BP 37, le Belvédère 1002, Tunis, Tunisia; e-mail: karim.saadaoui@isa2m.rnu.tn

** Department of Electrical and Electronics Engineering, Bilkent University, Bilkent Ankara TR-06800, Turkey; e-mail: ozguler@ee.bilkent.edu.tr

Recommended by Prof. Clarence W. de Silva

(paper no. 201-2030) regional pole placement is given. See also [5-11] for different methods of solving this problem. Recently, a method for determining the set of all proportional controllers that place the closed-loop poles in the region $S$ was given in [2].

The quest for an analytic design method for first-order controllers has been around for decades. Recently several computational methods have been proposed to determinethe set of all stabilizing first-order controllers. In [12, 13], stabilizing first-order controllers for continuous and discrete-time systems were determined using boundary crossing theorem to identify boundaries of stability region of two parameters, by sweeping over the third parameter the complete set can be determined. Using these results, it has been shown that it is possible to obtain $H_{\infty}$ optimal design with first-order controllers $[14,15]$. Alternative methods have been used to determine the total set of controllers' parameters that stabilize a given system. An exact solution to stabilizing discrete-time systems by firstorder controllers was given in [16]. Using extensions of the Hermite-Biehler theorem the set of all stabilizing firstorder controllers were determined in $[17,18]$. In this paper, we give a method to determine the set of all first-order controllers that place the poles of the closed-loop system in the region $S$. Once this set is determined, it is more convenient to search, among such controllers, those that satisfy other performance criteria imposed on the unit step response.

The paper is organized as follows. In Section 2, a generalization of the Hermite-Biehler theorem applicable to polynomials with complex coefficients is stated. This theorem is then used to convert the problem of determining gains such that a plant have a certain number of real roots to an equivalent problem of signature determination. In Section 3, we give an algorithm that solves the problem of determining all stabilizing first-order controllers that place the poles of the closed-loop system in a desired stability region. Section 4 contains some concluding remarks.

\section{A Generalization of the Hermite-Biehler Theo- rem}

In this section, a generalization of the Hermite-Biehler theorem to polynomials with complex coefficients [19] is 


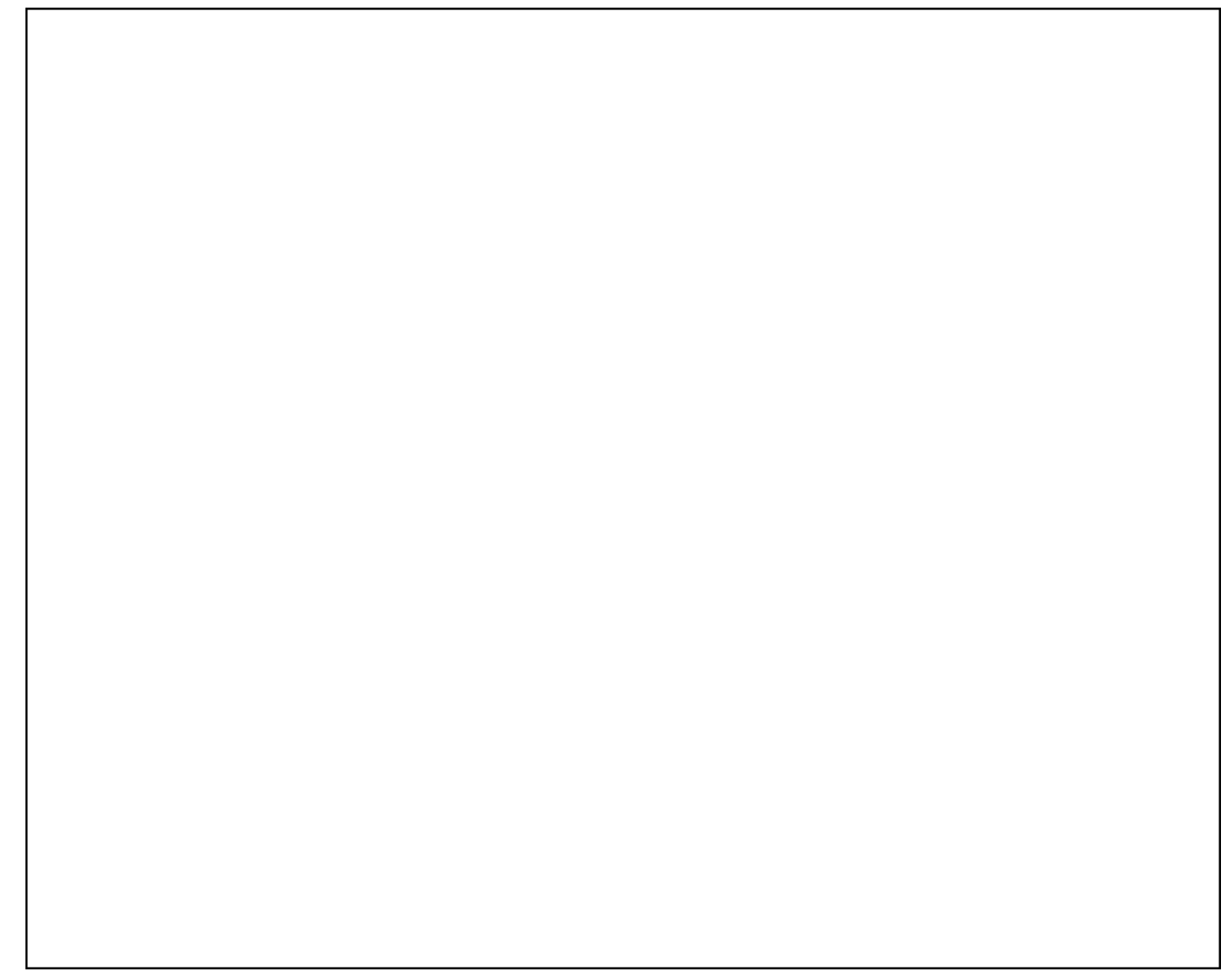

Figure 1. Stability region $S$.

presented. Before proceeding any further, let us fix the notation used in this paper. Let $\mathbf{R}$ denotes the set of real numbers and $\mathbf{C}$ denotes the set of complex numbers and let $\mathbf{C}_{-}, \mathbf{C}_{0}, \mathbf{C}_{+}$denote the points in the open left half, $j \omega$-axis, and the open right half of the complex plane, respectively. Given a set of polynomials $\psi_{1}, \ldots, \psi_{k} \in \mathbf{R}[s]$ not all zero and $k>1$, their greatest common divisor (with highest coefficient 1 ) is unique and it is denoted by $\operatorname{gcd}\left\{\psi_{1}, \ldots, \psi_{k}\right\}$. If $\operatorname{gcd}\left\{\psi_{1}, \ldots, \psi_{k}\right\},=1$, then we say $\left(\psi_{1}, \ldots, \psi_{k}\right)$ is coprime. The derivative of $\psi$ is denoted by $\psi^{\prime}$. The set $\mathcal{H}$ of Hurwitz stable polynomials are $\mathcal{H}=\left\{\psi(s) \in \mathbf{C}[s]: \psi(s)=0 \Rightarrow s \in \mathbf{C}_{-}\right\}$. The signature $\sigma(\psi)$ of a polynomial $\psi \in \mathbf{C}[s]$ is the difference between the number of its $\mathbf{C}_{-}$roots and $\mathbf{C}_{+}$roots. Given $\psi \in \mathbf{C}[s]$, the real and imaginary parts $(a, b)$ of $\psi(s)$ are the unique polynomials $a, b \in \mathbf{R}[\omega]$ such that $\psi(j \omega)=a(\omega)+j b(\omega)$. Finally, let us define the signum function $\mathcal{S}: \mathbf{R} \rightarrow\{-1,0,1\}$ by

$$
\mathcal{S} r=\left\{\begin{array}{c}
-1 \text { if } r<0 \\
0 \text { if } r=0 \\
1 \text { if } r>0
\end{array}\right.
$$

Theorem 1. [19] Let a non-zero polynomial $\psi \in \mathbf{C}[s]$ of degree $n$ have the real-imaginary parts $(a, b)$. Let $\omega_{1}<\omega_{2}<\cdots<\omega_{k}$ be the real, distinct finite roots of $b$ with odd multiplicities. Also let $\omega_{0}=-\infty, \omega_{k+1}=\infty$, and $\xi_{n}$ be the leading coefficient of $\psi(s)$. Then

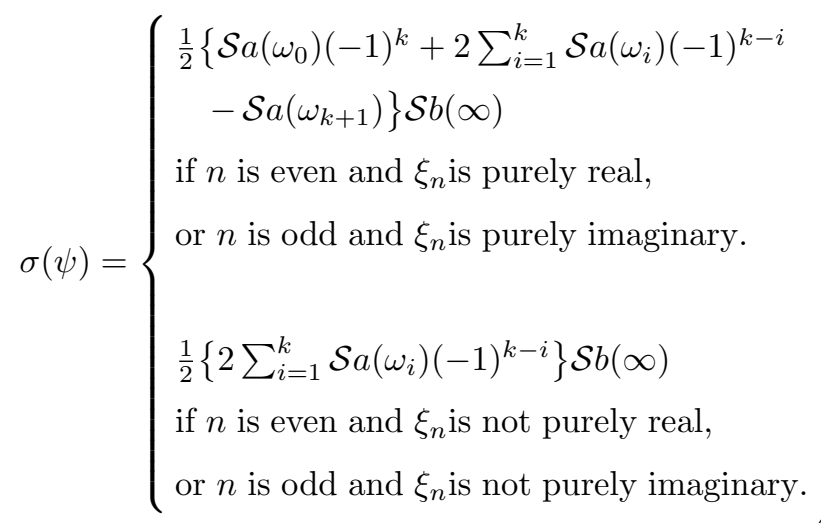

Proof: See $[2,19]$.

The following extension of Lemma 1 in [17] transforms the problem of determining the number of real roots of a real polynomial to an equivalent problem of finding the signature of a complex polynomial.

Lemma 1. A non-zero polynomial $\psi \in \mathbf{R}[u]$, has $r$ real roots without counting the multiplicities if and only if the signature of the complex polynomial $\bar{\psi}(j \omega)=\psi(w)+j \psi^{\prime}(w)$ is $-r$.

Proof: We first assume that $\left(\psi, \psi^{\prime}\right)$ is coprime. If $\operatorname{deg} \psi=n$, then $\operatorname{deg} \psi^{\prime}=n-1, \operatorname{deg} \bar{\psi}=n$, and the highest 
coefficient $\bar{\xi}_{n}$ of $\bar{\psi}(s)$ depends only on the highest coefficient $\xi_{n}$ of $\psi(\omega)$. If $n$ is even, then $(j \omega)^{n}$ is real. As $\xi_{n}=(j \omega)^{n} \bar{\xi}_{n}$ is real, it follows that $\bar{\xi}_{n}$ is real. If $n$ is odd, then $(j \omega)^{n}$ is imaginary and using similar arguments it follows that $\bar{\xi}_{n}$ is imaginary. In both cases, $n$ even or odd, we use the first equation of (1) in Theorem 1 to calculate the signature of $\bar{\psi}(s)$. Let $\psi(\omega)$ have $r$ real distinct roots $\omega_{1}<\omega_{2}<\cdots<\omega_{r}$. Since $\psi^{\prime}(w)$ is the derivative of $\psi(w)$, it follows that between any two consecutive real roots $\omega_{i}$ and $\omega_{i+1}$ of $\psi(\omega)$, there is an odd number of real roots of $\psi^{\prime}(\omega): v_{i 1}<v_{i 2}<\cdots<v_{i j}$, where $j$ is an odd integer. Since $\mathcal{S} \psi\left(v_{i 1}\right)=\mathcal{S} \psi\left(v_{i 2}\right)=\cdots=\mathcal{S} \psi\left(v_{i j}\right)$, it follows that $2 \mathcal{S} \psi\left(v_{i 1}\right)-2 \mathcal{S} \psi\left(v_{i 2}\right)+\cdots+(-1)^{j} 2 \mathcal{S} \psi\left(v_{i j}\right)=2 \mathcal{S} \psi\left(v_{i 1}\right)$. In the interval $\left(-\infty, \omega_{1}\right)$ or $\left(\omega_{r}, \infty\right), \psi^{\prime}(\omega)$ has an even number of real roots which do not affect the signature as the sign of $\psi$ is constant throughout the interval. Finally note that $\mathcal{S} \psi(\infty) \mathcal{S} \psi^{\prime}(\infty)=1, \ldots, \mathcal{S} \psi\left(v_{01}\right) \mathcal{S} \psi^{\prime}(\infty)=(-1)^{r-1}$, $\mathcal{S} \psi(-\infty) \mathcal{S} \psi^{\prime}(\infty)=(-1)^{r}$. Using these facts in (1) of Theorem 1, we get $\sigma(\bar{\psi})=\frac{1}{2}\left\{\mathcal{S} \psi(-\infty)(-1)^{r-1}+2 \mathcal{S} \psi\left(v_{01}\right)\right.$ $\left.(-1)^{r-2}+\cdots-\mathcal{S} \psi(\infty)\right\} \mathcal{S} \psi^{\prime}(\infty)=-r$. Therefore, by Theorem 1 , the signature of $\bar{\psi}(s)$ is $-r$. Conversely, let the signature of $\bar{\psi}(s)$ be $-r$. Using the first equation of (1) in Theorem 1, it follows that $\psi(\omega)$ changes sign exactly $r$ times. Hence, $\psi(\omega)$ has $r$ real roots. For non-coprime pair $\left(\psi, \psi^{\prime}\right)$, repeating similar arguments it is easy to prove that $\psi(\omega)$ has $r$ real roots without counting the multiplicities if and only if the signature of $\bar{\psi}(s)$ is $-r$.

We now briefly describe a method to determine constant stabilizing gains for complex polynomials. This method will be used in the next section. Given a proper plant $g(s)=p(s) / q(s)$, where $p, q \in \mathbf{C}[s]$ are coprime, the set $A_{r}(p, q):=\{\alpha \in \mathbf{R}: \quad \sigma[\phi(s, \alpha)]=\sigma[q(s)+\alpha p(s)]=r\}$ is the set of all real $\alpha$ such that $\phi(s, \alpha)$ has signature equal to $r$. Let $(h, g)$ and $(f, e)$ be the real-imaginary parts of $q$ and $p$, respectively, so that $q(j \omega)=h(\omega)+j g(\omega)$, $p(j \omega)=f(\omega)+j e(\omega)$. Let $d:=g c d\{f, e\}$ so that $f=d \bar{f}$, $e=d \bar{e}$, for coprime polynomials $\bar{f}, \bar{e} \in \mathbf{R}[\omega]$. Then, the polynomial $\bar{p}(s)$ such that $\bar{p}(j \omega):=\bar{f}(\omega)+j \bar{e}(\omega)$ is free of $\mathbf{C}_{0}$ roots. Let $m=\operatorname{deg} \bar{p}$ less than or equal to $n=\operatorname{deg} q$ and let $(H, G)$ be the real-imaginary parts of $q(s) \bar{p}^{*}(s)$ where $\bar{p}^{*}(j \omega):=\bar{f}(\omega)-j \bar{e}(j \omega)$. Also let $F(\omega):=p(s) \bar{p}^{*}(s)$. By a simple computation, it follows that,

$$
\begin{aligned}
H(\omega) & =h(\omega) \bar{f}(\omega)+g(\omega) \bar{e}(\omega) \\
G(\omega) & =g(\omega) \bar{f}(\omega)-h(\omega) \bar{e}(\omega) \\
F(\omega) & =f(\omega) \bar{f}(\omega)+e(\omega) \bar{e}(\omega)
\end{aligned}
$$

If $G \not \equiv 0$ and if they exist, let the real roots with odd multiplicities of $G(\omega)$ be $\left\{\omega_{1}, \ldots, \omega_{k}\right\}$ with the ordering $\omega_{1}<\omega_{2}<\cdots<\omega_{k}$, with $\omega_{0}:=-\infty$ and $\omega_{k+1}:=\infty$ for notational convenience and let $\xi$ be the leading coefficient of $[q(s)+\alpha p(s)] \bar{p}^{*}(s)$. The following algorithm determines whether $A_{r}(p, q)$ is empty or not and outputs its elements when it is not empty:

\section{Algorithm 1.}

\section{Calculate}

$$
\bar{\alpha}_{j}=\left\{\begin{array}{l}
-\frac{H}{F}\left(\omega_{j}\right), j=1, \ldots, k \& F\left(\omega_{j}\right) \neq 0, \\
\text { if } n+m \text { is even and } \xi \text { is not purely real, } \\
\text { or } n+m \text { is odd and } \xi \text { is not } \\
\text { purely imaginary. } \\
-\frac{H}{F}\left(\omega_{j}\right), j=0, \ldots, k+1 \& F\left(\omega_{j}\right) \neq 0, \\
\text { if } n+m \text { is even and } \xi \text { is purely real, } \\
\text { or } n+m \text { is odd and } \xi \text { is purely imaginary. }
\end{array}\right.
$$

and sort the distinct $\bar{\alpha}_{j}$ 's in ascending order

$$
\alpha_{0}<\alpha_{1}<\cdots<\alpha_{k+2}<\alpha_{k+3}
$$

where $\alpha_{0}=-\infty$ and $\alpha_{k+3}=\infty$.

2. Identify all the sequences of signums

$$
\mathcal{I}=\left\{\begin{aligned}
\left\{i_{1}, \ldots, i_{k}\right\} & \text { if } n+m \text { is even and } \xi \text { is } \\
& \text { not purely real, } \\
\text { or } n+m \text { is odd and } \xi \text { is } & \text { not purely imaginary. } \\
\left\{i_{0}, i_{1}, \ldots, i_{k+1}\right\} & \text { if } n+m \text { is even and } \xi \text { is } \\
& \text { purely real, } \\
\text { or } n+m \text { is odd and } \xi \text { is } & \text { purely imaginary. }
\end{aligned}\right.
$$

where $i_{j} \in\{-1,1\}$ for $j=0,1, \ldots, k+1$, that correspond to the intervals $\left(\alpha_{j}, \alpha_{j+1}\right)$ for $j=0, \ldots, k+2$. 3. For each signum sequence $\mathcal{I}_{j}$ from step 2 , if

$$
r+\sigma\left(p^{*}\right)=\left\{\begin{array}{l}
\left\{(-1)^{k-1} i_{0}+\cdots+i_{k-2}-i_{k-1}+i_{k}\right\} \\
\mathcal{S} G(\infty) \\
\text { if } n+m \text { is even and } \xi \text { is not } \\
\text { purely real, } \\
\text { or } n+m \text { is odd and } \xi \text { is not } \\
\text { purely imaginary. } \\
\frac{1}{2}\left\{(-1)^{k} i_{0}+\cdots-2 i_{k-1}+2 i_{k}-i_{k+1}\right\} \\
\mathcal{S} G(\infty) \\
\text { if } n+m \text { is even and } \xi \text { is } \\
\text { purely real, } \\
\text { or } n+m \text { is odd and } \xi \text { is } \\
\text { purely imaginary. }
\end{array}\right.
$$

holds, then $\left(\alpha_{j}, \alpha_{j+1}\right) \in A_{r}(p, q)$ 
Remark 1. By step 3 of Algorithm 1, a necessary condition for the existence of an $\alpha \in A_{r}(p, q)$ is that the imaginary part of $[q(s)+\alpha p(s)] \bar{p}^{*}(s)$ has at least $\left|r+\sigma\left(\bar{p}^{*}\right)\right|$ real roots with odd multiplicities if $n+m$ is even and $\xi$ is not purely real, or $n+m$ is odd and $\xi$ is not purely imaginary, and $\left|r+\sigma\left(\bar{p}^{*}\right)-1\right|$ real roots with odd multiplicities if $n+m$ is even and $\xi$ is purely real, or $n+m$ is odd and $\xi$ is purely imaginary.

\section{First-Order Controllers}

Given a plant $g(s)=p(s) / q(s)$ and a first-order controller $c(s)=\alpha_{2} s+\alpha_{3} / s+\alpha_{1}$, our objective is to find all values of $\left(\alpha_{1}, \alpha_{2}, \alpha_{3}\right)$ such that the closed-loop characteristic polynomial $\phi\left(s, \alpha_{1}, \alpha_{2}, \alpha_{3}\right)=\left(s+\alpha_{1}\right) q(s)+\left(\alpha_{2} s+\alpha_{3}\right) p(s)$ has all its roots in the region $S$ given in Fig. 1 . This is equivalent to solving three subproblems using the stability regions $S_{-\lambda}, S_{\theta}$, and $S_{-\theta}$ and finding the intersection of the solution sets.

Let us first solve the problem for the stability region $S_{\theta}$. Let us replace $s$ by $e^{j \theta} s$, then $\phi_{\theta}\left(s, \alpha_{1}, \alpha_{2}, \alpha_{3}\right)=\left(e^{j \theta} s+\alpha_{1}\right)$ $q\left(e^{j \theta} s\right)+\left(\alpha_{2} e^{j \theta} s+\alpha_{3}\right) p\left(e^{j \theta} s\right)$. Since $\theta$ is constant, we have $e^{j \theta}=\beta+j \gamma$ and $p, q \in C[s]$. The new characteristic polynomial is given by

$$
\begin{aligned}
\phi_{\theta}^{0}\left(s, \alpha_{1}, \alpha_{2}, \alpha_{3}\right)= & {\left[(\beta+j \gamma) s+\alpha_{1}\right] q(s) } \\
& +\left[\alpha_{2}(\beta+j \gamma) s+\alpha_{3}\right] p(s) \\
= & q_{0}(s)+\alpha_{3} p_{0}(s)
\end{aligned}
$$

where $\quad q_{0}(s)=\left[(\beta+j \gamma) s+\alpha_{1}\right] q(s)+\left[\alpha_{2}(\beta+j \gamma) s\right] p(s)$, $p_{0}(s)=p(s)$. Roots of $\phi\left(s, \alpha_{1}, \alpha_{2}, \alpha_{3}\right)$ in stability region $S_{\theta}$ is equivalent to roots of $\phi_{\theta}^{0}\left(s, \alpha_{1}, \alpha_{2}, \alpha_{3}\right)$ in the open left half complex plane. Using the generalized Hermite-Biehler theorem applicable to complex polynomials and Lemma 1, we describe in what follows a method to compute all values of $\left(\alpha_{1}, \alpha_{2}, \alpha_{3}\right)$ such that $\phi_{\theta}^{0}\left(s, \alpha_{1}, \alpha_{2}, \alpha_{3}\right)$ is Hurwitz stable. Recall that $q(j \omega)=h(\omega)+j g(\omega), p(j \omega)=f(\omega)+j e(\omega)$, $\bar{p}(j \omega)=\bar{f}(\omega)+j \bar{e}(\omega)$, and $q(j \omega) \bar{p}^{*}(j \omega)=H(\omega)+j G(\omega)$, $p(j \omega) \bar{p}^{*}(j \omega)=F(\omega)$, where $H, G$, and $F$ are given by $(2)$. Multiplying $\phi_{\theta}^{0}\left(j \omega, \alpha_{1}, \alpha_{2}, \alpha_{3}\right)$ by $\bar{p}_{0}^{*}(j \omega)$ we obtain

$$
\begin{aligned}
\psi_{\theta}^{1}\left(j \omega, \alpha_{1}, \alpha_{2}, \alpha_{3}\right)= & {\left[-\omega(\gamma H(\omega)+\beta G(\omega))+\alpha_{1} H(\omega)\right.} \\
& \left.-\alpha_{2} \omega \gamma F(\omega)+\alpha_{3} F(\omega)\right] \\
& +j[\omega(\beta H(\omega)-\gamma G(\omega)) \\
& \left.+\alpha_{1} G(\omega)+\alpha_{2} \omega \beta F(\omega)\right]
\end{aligned}
$$

Note that only two parameters $\left(\alpha_{1}, \alpha_{2}\right)$ appear in the imaginary part of $\psi_{\theta}^{1}(s)$. Suitable ranges of $\left(\alpha_{1}, \alpha_{2}\right)$ can be determined using Remark 1 and Lemma 1 as described below. The reasoning behind the algorithm which determines the set of parameters $\alpha_{1}, \alpha_{2}, \alpha_{3}$ of a stabilizing first-order controller can be explained as follows: suppose $\phi_{\theta}^{0}(s)$ is Hurwitz stable for some $\alpha_{1}, \alpha_{2}, \alpha_{3} \in$ R. By Remark 1, it follows that the imaginary part $\omega[\beta H(\omega)-\gamma G(\omega)]+\alpha_{1} G(\omega)+\alpha_{2} \omega \beta F(\omega)$ of $\psi_{\theta}^{1}(s)$ has at least $r_{1}=\left|n+1+\sigma\left(\bar{p}^{*}\right)\right|$ real roots with odd multiplicities. Suppose the imaginary part of $\psi_{\theta}^{1}(s)$ has $r_{1}$ real roots with odd multiplicities. By Lemma $1, \sigma\left[\phi_{\theta}^{1}(s)\right]=-r_{1}$, where

$$
\begin{aligned}
\phi_{\theta}^{1}(j \omega) & =H_{1}(j \omega)+\alpha_{1} G_{1}(j \omega)+\alpha_{2} F_{1}(j \omega) \\
& =q_{1}(j \omega)+\alpha_{2} p_{1}(j \omega)
\end{aligned}
$$

and $H_{0}(\omega)=\omega[\beta H(\omega)-\gamma G(\omega)], F_{0}(\omega)=\omega \beta F(\omega), G_{0}(\omega)=$ $G(\omega), H_{1}(j \omega)=H_{0}(\omega)+j H_{0}^{\prime}(\omega), F_{1}(j \omega)=F_{0}(\omega)+j F_{0}^{\prime}(\omega)$, $G_{1}(j \omega)=G_{0}(\omega)+j G_{0}^{\prime}(\omega), \quad q_{1}(s)=H_{1}(s)+\alpha_{1} G_{1}(s)$, and $p_{1}(s)=F_{1}(s)$. To find the suitable ranges of $\alpha_{1}$ and $\alpha_{2}$, we modify $\phi_{\theta}^{1}(s)$ as follows: let $B:=\operatorname{gcd}\left\{F_{0}, F_{0}^{\prime}\right\}$ so that $F_{0}=B \bar{F}_{0}, F_{0}^{\prime}=B \bar{F}_{0}^{\prime}$ for coprime polynomials $\bar{F}_{0}, \bar{F}_{0}^{\prime} \in \mathbf{R}[w]$. Also let $\bar{p}_{1}(j \omega):=\bar{F}_{0}(\omega)+j \bar{F}_{0}^{\prime}(\omega)$. By a simple computation, it follows that,

$$
\begin{aligned}
\psi_{\theta}^{2}\left(j \omega, \alpha_{1}, \alpha_{2}\right)= & \phi_{\theta}^{1}\left(j \omega, \alpha_{1}, \alpha_{2}\right) \bar{p}_{1}^{*}(j \omega) \\
= & H_{2 r}(\omega)+\alpha_{1} G_{2 r}(\omega)+\alpha_{2} F_{2 r}(\omega) \\
& +j\left[H_{2 i}(\omega)+\alpha_{1} G_{2 i}(\omega)\right]
\end{aligned}
$$

where

$$
\begin{aligned}
& H_{2 r}(\omega)=H_{0}(\omega) \bar{F}_{0}(\omega)+H_{0}^{\prime}(\omega) \bar{F}_{0}^{\prime}(\omega), \\
& F_{2 r}(\omega)=F_{0}(\omega) \bar{F}_{0}(\omega)+F_{0}^{\prime}(\omega) \bar{F}_{0}^{\prime}(\omega), \\
& G_{2 r}(\omega)=G_{0}(\omega) \bar{F}_{0}(\omega)+G_{0}^{\prime}(\omega) \bar{F}_{0}^{\prime}(\omega), \\
& H_{2 i}(\omega)=H_{0}^{\prime}(\omega) \bar{F}(\omega)-H_{0}(\omega) \bar{F}_{0}^{\prime}(\omega), \\
& G_{2 i}(\omega)=G_{0}^{\prime}(\omega) \bar{F}_{0}(\omega)-G_{0}(\omega) \bar{F}_{0}^{\prime}(\omega) .
\end{aligned}
$$

Now only one parameter $\alpha_{1}$ appears in the imaginary part of $\psi_{\theta}^{2}(s)$. Once more by Remark 1, since $\sigma\left[\phi_{\theta}^{1}(s) \bar{p}_{1}^{*}(s)\right]=-r_{1}+\sigma\left[\bar{p}_{1}^{*}(s)\right]$ the imaginary part of $\phi_{\theta}^{1}(s) \bar{p}_{1}^{*}(s)$ should have at least $r_{2}=\left|-r_{1}+\sigma\left(\bar{p}_{1}^{*}\right)\right|$ real roots with odd multiplicities . Now the set of $\alpha_{1} \in \mathbf{R}$ which achieves $r_{2}$ real roots with odd multiplicities in $H_{2 i}(\omega)+\alpha_{1} G_{2 i}(\omega)$ can be determined by applying Algorithm 1 to $q_{2}(j \omega)=H_{2}(j \omega)=H_{2 i}(\omega)+j H_{2 i}^{\prime}(\omega)$ and $p_{2}(j \omega)=G_{2}(j \omega)=G_{2 i}(\omega)+j G_{2 i}^{\prime}(\omega)$. In each step, we eliminate one of the controller's parameters and determine conditions to find the remaining ones. The algorithm below traces the above steps backwards by repetition of the following steps (i)-(iii):

(i) Pick a value of $\alpha_{1}$ such that the number of real roots with odd multiplicities of $H_{2 i}(\omega)+\alpha_{1} G_{2 i}(\omega)$ is $r_{2}$ or greater.

(ii) Determine using Algorithm 1 all $\alpha_{2} \in \mathbf{R}$ such that $\sigma\left[\phi_{\theta}^{1}(s)\right]=-r_{1}$. This is equivalent to determining values of $\alpha_{2}$ such that $H_{0}(\omega)+\alpha_{1} G_{0}(\omega)+\alpha_{2} F_{0}(\omega)$ has $r_{1}$ real roots with odd multiplicities.

(iii) For every $\alpha_{2}$ determined, find using Algorithm 1 again, all $\alpha_{3}$ such that $\phi_{\theta}^{0}(s)$ is Hurwitz stable.

\section{Algorithm 2.}

1. Partition the real axis into intervals (or union of intervals) such that the number of real roots with odd multiplicities of $H_{2 i}(\omega)+\alpha_{1} G_{2 i}(\omega)$ is constant in each interval.

2. $F i x r_{1}=\left|n+\sigma\left(\bar{p}_{0}^{*}\right)+1\right|$.

(a) Find admissible ranges of $\alpha_{1}$ from the intervals found in the first step. 
i. Fix an $\alpha_{1}$ in the admissible range.

ii. Apply Algorithm 1 to $q_{1}(s)$ and $p_{1}(s)$. (This calculates admissible values of $\alpha_{2}$ such that $H_{0}(\omega)+\alpha_{1} G_{0}(\omega)+\alpha_{2} F_{0}(\omega)$ has $r_{1}$ real roots with odd multiplicities.)

A. Fix an $\alpha_{2}$ from the range determined in 2.a.ii.

B. Apply Algorithm 1 to $q_{0}(s)$ and $p_{0}(s)$. (This calculates all admissible values of $\alpha_{3}$ such that $\phi_{\theta}^{0}$ is in $\mathcal{H}$.)

C. Increment $\alpha_{2}$ and go to step 2.a.ii.B.

iii. Increment $\alpha_{1}$ and go to step 2.a.ii.

(b) Increment $r_{1}$ and go to step 2.a.

For the stability region $S_{-\theta}$, it was shown in [2] for the case of proportional controllers, that $S_{-\theta}$ and $S_{\theta}$ have exactly the same set of stabilizing controllers. This conclusion holds for first-order controllers. To see this, suppose that for a given triplet $\left(\bar{\alpha}_{1}, \bar{\alpha}_{2}, \bar{\alpha}_{3}\right), s_{0}$ is a root of $\phi\left(s, \alpha_{1}, \alpha_{2}, \alpha_{3}\right)$, then $\left(e^{j \theta} s_{0}+\bar{\alpha}_{1}\right) q\left(e^{j \theta} s_{0}\right)+\left(\bar{\alpha}_{2} e^{j \theta} s_{0}+\bar{\alpha}_{3}\right)$ $p\left(e^{j \theta} s_{0}\right)=0$. As $q(s)$ and $p(s)$ are real polynomials, it follows that $\left(e^{-j \theta} s_{0}^{*}+\bar{\alpha}_{1}\right) q\left(e^{-j \theta} s_{0}^{*}\right)+\left(\bar{\alpha}_{2} e^{-j \theta} s_{0}^{*}+\bar{\alpha}_{3}\right)$ $p\left(e^{-j \theta} s_{0}^{*}\right)=0$ where $s_{0}^{*}$ is the complex conjugate of $s_{0}$. Since $s_{0}^{*}$ and $s_{0}$ have the same real part, it follows that $\left(\bar{\alpha}_{1}, \bar{\alpha}_{2}, \bar{\alpha}_{3}\right)$ is stabilizing triplet for the stability region $S_{-\theta}$ if and only if it is stabilizing triplet for the stability region $S_{\theta}$.

Now let us consider the problem of determining the stabilizing values of $\left(\alpha_{1}, \alpha_{2}, \alpha_{3}\right)$ for the shifted Hurwitz stability region $S_{-\lambda}$. Let us replace $s$ by $s-\lambda$ and make the corresponding changes. We now solve the usual stabilization problem for the new characteristic polynomial $\phi_{\lambda}\left(s, \alpha_{1}, \alpha_{2}, \alpha_{3}\right)$. As we are using a dynamic controller, the new characteristic polynomial is given by $\phi_{\lambda}\left(s, \alpha_{1}, \alpha_{2}, \alpha_{3}\right)=\left(s+\alpha_{1}-\lambda\right) q(s)+\left(\alpha_{2} s+\alpha_{3}-\alpha_{2} \lambda\right) p(s)$. Multiplying $\phi_{\lambda}\left(s, \alpha_{1}, \alpha_{2}, \alpha_{3}\right)$ by $\bar{p}(-s)$ we obtain

$$
\begin{aligned}
\psi_{\lambda}\left(s, \alpha_{1}, \alpha_{2}, \alpha_{3}\right)= & s^{2} G\left(s^{2}\right)-\lambda H\left(s^{2}\right)+\alpha_{1} H\left(s^{2}\right) \\
& -\alpha_{2} \lambda F\left(s^{2}\right)+\alpha_{3} F\left(s^{2}\right)+s\left[H\left(s^{2}\right)\right. \\
& \left.-\lambda G\left(s^{2}\right)+\alpha_{1} G\left(s^{2}\right)+\alpha_{2} F\left(s^{2}\right)\right]
\end{aligned}
$$

We can use the method described above to find stabilizing values of $\left(\alpha_{1}, \alpha_{2}, \alpha_{3}\right)$. In [18], an alternative method that take advantage of the fact that $\psi_{\lambda}(s)$ is a real polynomial was given.

Example 1. Consider a first-order controller to stabilize the unstable plant $g(s)=p(s) / q(s)$ where $q(s)=s^{5}+3 s^{4}+$ $29 s^{3}+15 s^{2}-3 s+60, \quad p(s)=s^{3}-6 s^{2}+2 s-1$, and the stability region $S$ is the intersection of two rotated stability regions $S_{\pi / 18}$ and $S_{-\pi / 18}$. Let us replace $s$ by $e^{j \pi / 18} s$, then $q(s)=(0.6428+j 0.7660) s^{5}+(2.2981+j 1.9284) s^{4}+$ $(25.1147+j 14.5000) s^{3}+(14.0954+j 5.1303) s^{2}-(2.9544+$ $j 0.5209) s+60, \quad p(s)=(0.8660+j 0.5000) s^{3}-(5.6382+$ $j 2.0521) s^{2}+(1.9696+j 0.3473) s-1$. Using Algorithm 2, the stabilizing values of $\left(\alpha_{1}, \alpha_{2}, \alpha_{3}\right)$ are obtained as shown in Fig. 2.

Remark 2. This method can be applied to PID controllers. Let $c(s)=\left(\alpha_{1} s^{2}+\alpha_{2} s+\alpha_{3}\right) / s$, then we obtain

$$
\begin{aligned}
\psi_{\lambda}\left(s, \alpha_{1}, \alpha_{2}, \alpha_{3}\right)= & {\left[s^{2} G\left(s^{2}\right)-\lambda H\left(s^{2}\right)+\alpha_{1} s^{2} F\left(s^{2}\right)\right.} \\
& \left.+\alpha_{1} \lambda^{2} F\left(s^{2}\right)-\alpha_{2} \lambda F\left(s^{2}\right)+\alpha_{3} F\left(s^{2}\right)\right] \\
& +s\left[H\left(s^{2}\right)-\lambda G\left(s^{2}\right)-\alpha_{1} 2 \lambda F\left(s^{2}\right)\right. \\
& \left.+\alpha_{2} F\left(s^{2}\right)\right]
\end{aligned}
$$

Figure 2. Stabilizing values $\left(\alpha_{1}, \alpha_{2}, \alpha_{3}\right)$. 


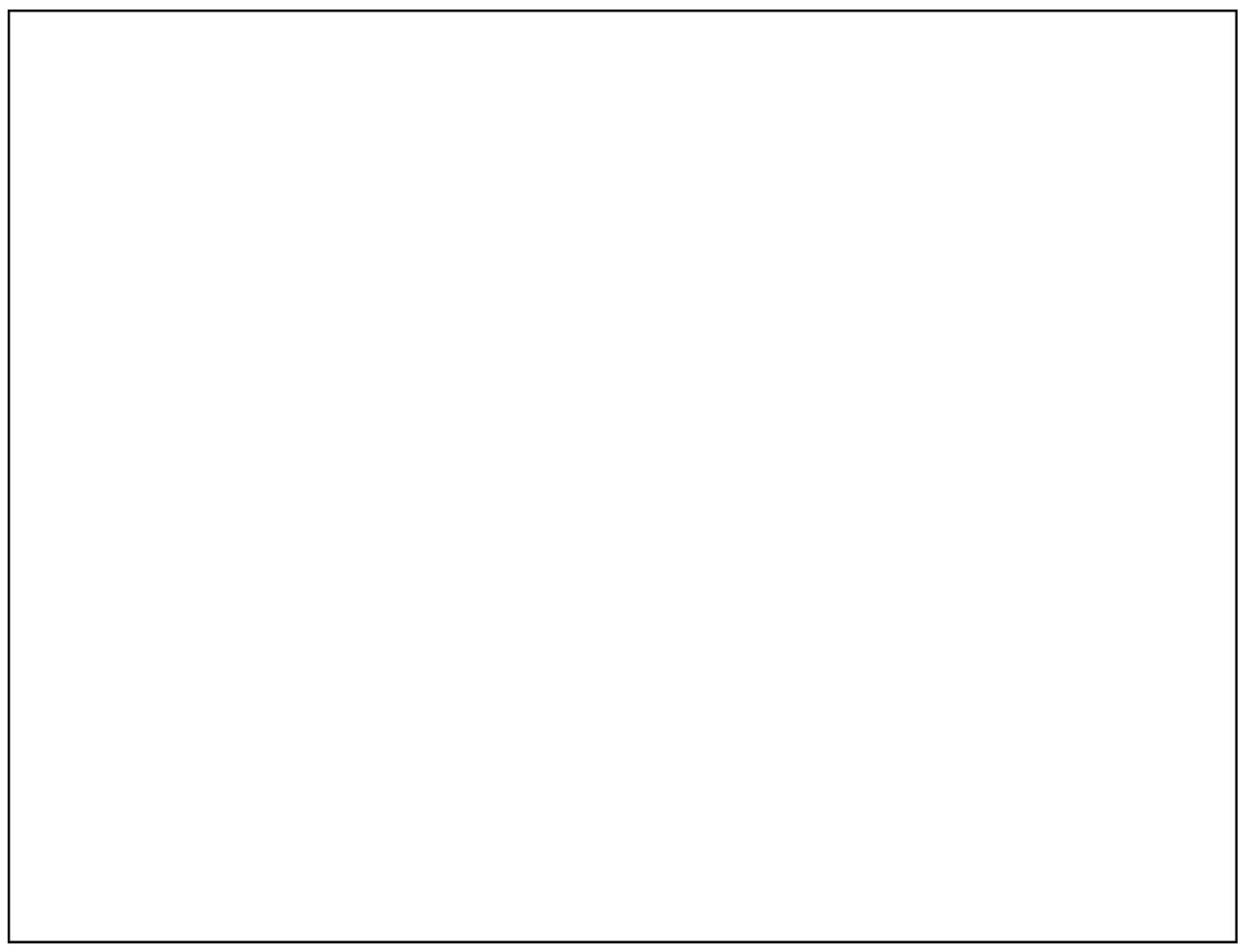

Figure 3. Stabilizing values $\left(\alpha_{1}, \alpha_{2}\right)$.

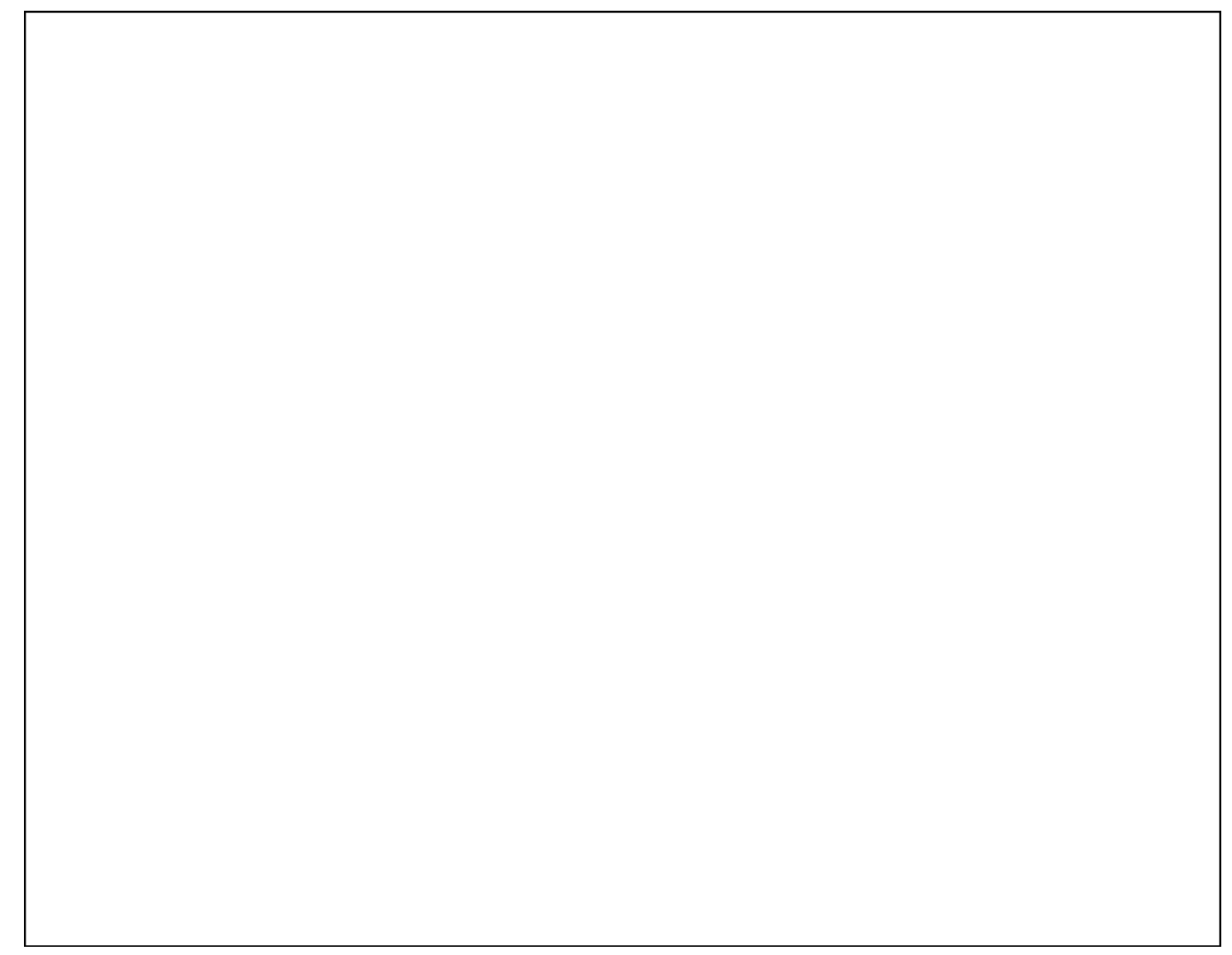

Figure 4. Attainable roots with respect to region $S$ for $\alpha_{1}=-0.7599$. 
and

$$
\begin{aligned}
\psi_{\theta}\left(j \omega, \alpha_{1}, \alpha_{2}, \alpha_{3}\right)= & {\left[-\omega\left(\gamma H_{0}(\omega)+\beta G_{0}(\omega)\right)-\alpha_{1} \omega^{2}\right.} \\
& \left(\beta^{2}-\gamma^{2}\right) F_{0}(\omega)-\alpha_{2} \omega \gamma F_{0}(\omega) \\
& \left.+\alpha_{3} F_{0}(\omega)\right]+j\left[\omega\left(\beta H_{0}(\omega)-\gamma G_{0}(\omega)\right)\right. \\
& \left.-\alpha_{1} \omega^{2} 2 \beta \gamma F_{0}(\omega)+\alpha_{2} \omega \beta F_{0}(\omega)\right]
\end{aligned}
$$

As two parameters $\left(\alpha_{1}, \alpha_{2}\right)$ appear in the odd part of $\psi_{\lambda}\left(s, \alpha_{1}, \alpha_{2}, \alpha_{3}\right)$, imaginary part of $\psi_{\theta}\left(s, \alpha_{1}, \alpha_{2}, \alpha_{3}\right)$, we can directly apply the method developed for first-order controllers.

Example 2. Consider a PI controller $c(s)=\left(\alpha_{1} s+\alpha_{2}\right) / s$ to stabilize the plant $g(s)=p(s) / q(s)$ given in [2], where $q(s)=s^{3}+3 s^{2}+4 s, p(s)=s^{2}+2 s-2$. The stability region $S$ is given in Fig. 1 and specified by the parameters $\gamma=0.5$ and $\theta=\pi / 6$. For the rotated Hurwitz stability regions $S_{\theta}$ and $S_{-\theta}$, replacing $s$ by $s e^{j \pi / 6}$, we get $q(s)=j s^{3}+(1.5+2.5981 j) s^{2}+(3.4641+2 j) s$, $p(s)=(0.5+0.866 j) s^{2}+(1.7321+j) s-2$. For the shifted Hurwitz stability region $S_{-\gamma}$, replacing $s$ by $s-0.5$ we get $q(s)=s^{3}+1.5 s^{2}+1.75 s-1.375, \quad p(s)=s^{2}+s-2.75$. Using these new polynomials and the method described in this section, we obtain the stabilizing values of $\left(\alpha_{1}, \alpha_{2}\right)$ as shown in Fig. 3. Let $\alpha_{1}=-0.7599$, then $(-0.1489,-0.13)$ is the stabilizing interval for $\alpha_{2}$. To check the results obtained, the root-locus for the values of $\alpha_{2}$ in this interval is shown in Fig. 4 and clearly these roots belong to the stability region $S$.

\section{Conclusions}

This paper gives a computational method to determine the set of all first-order controllers that place the poles of the closed-loop system in a sector of the left-half plane. The computation is based on a generalization of the HermiteBiehler theorem applicable to complex polynomials. Since this method is based on eliminating one of the controller's parameters, at each step, and determining conditions to find the remaining ones, extension of this method to controllers with more than three parameters is possible.

\section{References}

[1] E.D. Sontag, Mathematical control theory: Deterministic finite dimensional systems Second Edition (New York: Springer, 1998).

[2] A. Datta, M.T. Ho, \& S.P. Bhattacharyya, Structure and synthesis of PID controllers (New York: Springer-Verlag, 2000).

[3] M. Vidyasagar, Control system synthesis: a factorization approach (Cambridge, Massachussets: MIT press, 1985).

[4] W.M. Haddad \& D.S. Bernstein, Controller design with regional pole constraints, IEEE Transactions on Automatic Control, 37(1), 1992, 54-69.

[5] A.A. Abdul-Wahab \& M.A. Zohdy, Eigenvalue clustering in subregions of the complex plane, International Journal of Control, 48, 1988, 2527-2538.

[6] A.A. Mohammed, A new optimal root-locus technique fro LQR design, Control and Intelligent Systems, 35(1), 2007.

[7] M. Benrejeb, P. Borne, \& F. Laurent, Sur une application de la forme en flèche à l'analyse des processus, RAIRO Automatique, 16(2), 1982, 133-146.
[8] P. Borne \& M. Benrejeb, On the representation and the stability study of large scale systems, International Journal of Computers, Communications \& Control, Supplementary Issue: Proceedings of ICCCC2008, 3, 2008, 55-66.

[9] Y.J. Pan \& J. Gu, Remote stabilization of a class of linear systems and its robust stability analysis, Control and Intelligent Systems, 35(1), 2007.

[10] L.S. Shieh, H.M. Dib, \& S. Ganesan, Linear quadratic regulators with eigenvalue placement in a specified region, Automatica, 24, 1988, 819-823.

[11] B. Wittenmark, R.J. Evans, \& Y.C. Soh, Constrained pole placement using transformation and LQ-design, Automatica, 23, 1987, 767-769.

[12] R.N. Tantaris, L.H. Keel, \& S.P. Bhattacharyya, Stabilization of continuous time systems by first-order controllers, Proc. 10th Mediterranean Conference on Control and Automation, Lisbon, Portugal, 2002.

[13] R.N. Tantaris, L.H. Keel, \& S.P. Bhattacharyya, Stabilization of discrete-time systems by first-order controllers, IEEE Transactions on Automatic Control, 48(5), 2003, 858-860.

[14] R.N. Tantaris, L.H. Keel, \& S.P. Bhattacharyya, $H_{\infty}$ design with first-order controllers, Proc. 42nd IEEE Conference on Decision and Control, Hawii, USA, 2003, 2276-2281.

[15] R.N. Tantaris, L.H. Keel, \& S.P. Bhattacharyya, $H_{\infty}$ design with first-order controllers, IEEE Transactions on Automatic Control 51(8), 2006, 1343-1347.

[16] P. Yu \& Z. Wu, An exact solution to the stabilization of discrete systems using a first-order controller, IEEE Transactions on Automatic Control 50(9), 2005, 1375-1379.

[17] K. Saadaoui \& A.B. Özgüler, On the set of all stabilizing firstorder controllers, Proc. American Control Conference ACC'03, Denver, Colorado, 2003.

[18] K. Saadaoui \& A.B. Özgüler, A new method for the computation of all stabilizing controllers of a given order, International Journal of Control, 78(1), 2005, 14-28.

[19] M.T. Ho, Synthesis of $H_{\infty}$ PID controllers: A parametric approach, Automatica, 39, 2003, 1069-1075.

\section{Biographies}

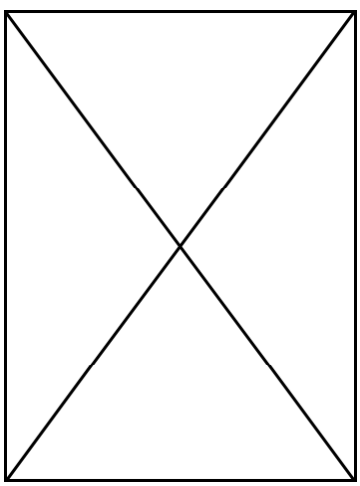

Karim Saadaoui received his $\mathrm{PhD}$ at the Electrical and Electronics Engineering Department of the University of Bilkent, Ankara in 2003. He is a researcher at the Research unit LA.R.A. Automatique of the Engineering School of Tunis ENIT, Tunisia. Dr. Saadaoui research interests are in the areas of time delay systems, stability robustness, and applications of robust control theory to process control problems.

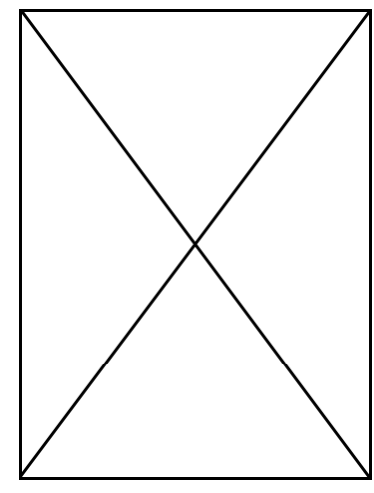

A. Bülent Özgüler received his $\mathrm{PhD}$ at the Electrical Engineering Department of the University of Florida, Gainesville in 1982. He was a researcher at the Marmara Research Institute of TÜBITAK during 1983-1986. He spent one year at the Institut für Dynamische Systeme, Bremen Universität, Germany, on Alexander von Humboldt Scholarship during 1994-1995. He is with the Electrical and Electronics Engineering Department of Bilkent 
University, Ankara since 1986. Prof. Özgüler's research interests are in the areas of decentralized control, stability robustness, realization theory, linear matrix equations, and application of system theory to social sciences. He has about 60 research papers in the field and is the author of the book Linear Multichannel Control: A System Matrix Approach, Prentice Hall, 1994. 